\title{
Virtual reality exposure-based therapy as an emerging treatment modality for anxiety disorders
}

Wendy Wang, Sean Wong

\begin{abstract}
Anxiety disorders are the most prevalent mental health condition, affecting one-third of the population during their lifetime. Patient with anxiety may experience overwhelming fear to an irrational fear that can impair everyday functioning. Current treatment for anxiety disorders include pharmacological (i.e. selective serotonin reuptake inhibitors) and psychological (i.e. cognitive behavioural therapy) intervention. Cognitive behavioural therapy is an effective exposure-based therapy utilizing repetitive exposure to the feared stimulus to develop desensitization and tolerance, but holds high dropout rates due to unbearable anxiety for patients. Recognizing this challenge, virtual reality technology is emerging as a promising tool for patients to challenge their fear in a simulated environment based on individual progression. This article explores the new development of virtual reality technology as an effective treatment modality for anxiety disorders to enhance current approaches in mental health care.
\end{abstract}

\section{INTRODUCTION}

Anxiety disorders, including panic disorder with or without agoraphobia, generalized anxiety disorder, social anxiety disorder, specific phobias, and separation anxiety disorder, are the most prevalent mental health conditions, with up to $33.7 \%$ of the Canadian population affected during their lifetime. ${ }^{1}$ Patients with anxiety experience overwhelming fear to perceived danger. As a result, they adapt avoidance and safety behaviours which can impair social functioning. Despite the widespread prevalence of anxiety disorders, they are largely unrecognized and undertreated. ${ }^{1}$ Treatments for anxiety disorders are effective, with pharmacological and psychotherapy interventions, and are commonly managed in the primary care setting. However, patients with severe anxiety may have limited access to resources offered by psychiatric specialists or may be unresponsive to conventional therapy. ${ }^{1}$ Recognizing this challenge, virtual reality (VR) technology emerges as a potential tool to enhance current approaches to mental health care. This article will explore the new developments of VR technology in exposure-based intervention as an effective treatment modality for anxiety disorders.

\section{CURRENT TREATMENT FOR ANXIETY DISORDERS}

Anxiety disorders can be effectively treated with psychological or pharmacological interventions. ${ }^{2}$ Current guidelines recommend utilizing either cognitive behavioral therapy (CBT) or antidepressant medication, such as selective serotonin reuptake inhibitors (SSRIs). ${ }^{2}$
Although greater empirical evidence supports CBT, both SSRIs and CBT are first-line treatment modalities for anxiety disorders. The choice of therapy is based on patient preference, but more patients choose SSRIs over CBT treatment as medications are less expensive and more accessible in the primary care setting. ${ }^{2}$ Considering that anxiety symptoms are maintained with avoidance safety behaviours in a positive feedback loop, treatment requires repetitive exposure to anxiety-inducing stimulus to change irrational beliefs and develop adaptive coping mechanisms to stress. With gradual exposure to the stimulus, desensitization will occur without the need to engage in avoidance behaviours. ${ }^{2}$ Exposure-based therapy is a gradual approach that can be categorized into imaginal, in vitro, and in vivo exposures. Patients first imagine experiencing the anxiety-inducing trigger. Then, exposure is achieved through images, simulated stimulus, or virtual reality. Lastly, in vivo exposure requires the patient to face the fear inducing stimulus in real life, which has high dropout rates as patients cannot tolerate the anxiety. Thus, VR could be useful for patients to challenge their fear in a simulated environment through repeated practice, coaching, and support by the clinician prior to transitioning to in vivo exposure therapy.

\section{VR TECHNOLOGY OVERVIEW \& APPLICATIONS IN PSYCHOLOGICAL THERAPY}

VR technology has been increasingly incorporated into mental health treatment and clinical research. VR creates a computergenerated three-dimensional environment that allows patients to feel a sense of immersion. ${ }^{3}$ Users can interact with the immersive virtual environment by wearing motion sensors and a headmounted display that provides auditory and visual feedback. VR is a potentially powerful treatment tool in psychiatry as the experience of the patient can be repeated and modified within a safe environment. Additionally, VR therapy aims to parallel reality with precise control of sensory cues to increase memories of behaviour modification and real-life applicability. ${ }^{3}$ VR exposure therapy has been shown as an effective treatment tool for many mental health disorders. Notably, VR technology in the treatment for anxiety disorder has the strongest evidence-based support compared to other psychiatric disorders. Specifically, five separate clinical trials for VR exposure therapy have been published for treating social anxiety disorder, four studied panic disorder, two studied fear of flying, and two studied spider phobia. ${ }^{3}$ VR technology has also been utilized in treating smoking addiction, pain, post-traumatic stress disorder, eating disorders, and schizophrenia, as well as social cognition training for children with high functioning autism to improve emotion recognition, social attribution, and analogical reasoning., ${ }^{4,5}$ 


\section{TREATMENT COURSE FOR VR EXPOSURE THERAPY}

As a component of CBT, VR exposure therapy focuses on modifying the patient's cognitive and behavioural response to the fear stimulus with repeated exposure in order to reduce anxiety. This typically begins with an introduction session focused on explaining the rationale for exposure, recognizing safety behaviours, and identifying possible coping strategies. The content for VR exposure is often personalized and the progression plan is determined by thorough assessment of the patient's fears. Patients are then immersed in the VR-simulated environment and interact with the anxiety-inducing stimulus. Repetitive exposure to the stimulus will allow patients to become increasingly desensitized and develop sufficient tolerance towards that fear. ${ }^{3}$ VR exposure therapy allows patients to progress at an individualized pace through an exposure hierarchy with increasing difficulty. For example, the hierarchy for fear of flying is broken down into steps such as walking through the airport terminal, take-off and landing, and flying during a thunderstorm. Patients can repeat any level of the hierarchy until anxiety symptoms are manageable before progressing forward to the next step. The delivery of sensory information is highly controlled by the clinician, who also monitors the patient's distress and behaviour throughout the experience. The clinician can encourage and facilitate emotional engagement of the patient within VR while preventing avoidance behaviours. ${ }^{3}$ Treatment progression based on readiness of the patient promotes collaboration in the therapeutic relationship.

\section{BENEFITS OF VR EXPOSURE}

The benefits of VR exposure therapy in treating anxiety disorders have been supported by a wide body of research. Firstly, VR exposure therapy is shown to be more effective than conventional imaginal exposure therapy and as effective as in vivo exposure therapy. The immersive VR environment triggers the same feeling of anxiety, but being aware that the stimuli is not real allows them to challenge their fears without real-life consequences. This provides a stepping stone for patients that cannot yet tolerate direct exposure to anxiety-inducing stimuli to decrease dropout from treatment. ${ }^{3}$ Additionally, VR exposure is more cost-effective and practical compared to CBT in vivo exposure therapy since any environment can be replicated within treatment. ${ }^{6}$ For example, VR exposure therapy for the fear of flying allows both the patient and therapist avoid significant time commitments and cost of repetitive use of airplanes. Fear of heights similarly require travel to tall buildings that may be limited based on location. Significant behaviour changes learned during VR exposure therapy is comparable to in vivo methods and carry over to real-life situations.

\section{DRAWBACKS AND LIMITATIONS}

While most clinical studies demonstrate that VR exposure therapy is comparable to standard CBT treatment, it is important to recognize that preliminary studies utilized small samples and lacked comparison groups, which emphasizes the need for more rigorously controlled studies. Future research is also needed to identify the modifying factors of VR-based treatment on outcome, such as the therapeutic relationship and engagement during exposure. ${ }^{3}$ Furthermore, there are limits in the clinical application of VR technologies. VR exposure therapy requires extensive hardware to deliver a high-quality immersive experience, which may increase cost and decrease its availability. The lack of regulatory systems for mobile technologies makes the quality of delivered treatment difficult to monitor. Additionally, there are concerns that the sensory stimulation during VR exposure therapy may trigger headaches, seizures, and gait abnormalities in susceptible individuals. Most importantly, although VR technology have been thoroughly developed in the market and research studies have been completed, its use in clinical practice is still fairly novel due to lack of availability to VR hardware and software. Some platforms are beginning to adopt evidence-based protocols to provide standardized care, but the extensive incorporation of VR exposure therapy in clinical settings would require further research.

\section{CONCLUSION}

VR exposure therapy holds promise as an emerging tool for the treatment for anxiety disorders as well as other psychiatric conditions. A wide body of literature exists detailing how VR exposure therapy provides lasting behavioral modifications that is applicable to real-life situations. It is important to recognize that VR treatment is only one component of psychotherapy; a trusting therapeutic relationship between the clinician and patient remains key to reach treatment targets. More health care efforts need to focus on delivering efficacious mental health treatment, education, and prevention, particularly in primary health settings. As VR technology becomes compatible with smart phone applications, VRbased treatments for mental health will become more accessible. The future direction of VR technology holds great potential in enhancing the care for patients with anxiety disorders.

\section{REFERENCES}

1. Bandelow B, Michaelis S. Epidemiology of anxiety disorders in the 21st century. Dialogues Clin Neurosci. 2015 Sep;17(3):327-35.

2. Bystritsky A, Khalsa SS, Cameron ME, et al. Current diagnosis and treatment of anxiety disorders. P T. 2013 Jan;38(1):30-57.

3. Maples-Keller JL, Bunnell BE, Kim SJ, et al. The use of virtual reality technology in the treatment of anxiety and other psychiatric disorders. Harv Rev Psychiatry. 2017 May/Jun;25(3):103-13. https://doi.org/10.1097/HRP.0000000000000138

4. Didehbani N, Allen T, Kandalaft M, et al. Virtual reality social cognition training for children with high functioning autism. Comput Human Behav. 2016 Sep;62:703-11. https://doi.org/10.1016/j.chb.2016.04.033

5. Valmaggia LR, Latif L, Kempton MJ et al. Virtual reality in the psychological treatment for mental health problems: an systematic review of recent evidence. Psychiatry Res. 2016 Feb;236:189-95. https://doi.org/10.1016/j.psychres.2016.01.015

6. Bouchard S, Dumoulin S, Robillard G. et al. Virtual reality compared with in vivo exposure in the treatment of social anxiety disorder: a three-arm randomised controlled trial. Br J Psychiatry. 2017 Apr;210(4):276-83. https://doi.org/10.1192/bjp.bp.116.184234

7. Morina N, Ijntema H, Meyerbröker K, et al. Can virtual reality exposure therapy gains be generalized to real-life? A meta-analysis of studies applying behavioral assessments. Behav Res Ther. 2015 Nov;74:18-24. https://doi.org/10.1016/j.brat.2015.08.010 